\title{
Mappings of Type Special Space of Sequences
}

\author{
Awad A. Bakery ${ }^{1,2}$ \\ ${ }^{1}$ Department of Mathematics, Faculty of Science and Arts, University of Jeddah (UJ), P.O. Box 355, Khulais 21921, Saudi Arabia \\ ${ }^{2}$ Department of Mathematics, Faculty of Science, Ain Shams University, P.O. Box 1156, Abbassia, Cairo 11566, Egypt
}

Correspondence should be addressed to Awad A. Bakery; awad_bakery@yahoo.com

Received 12 April 2016; Revised 26 June 2016; Accepted 28 July 2016

Academic Editor: Gianluca Vinti

Copyright (C) 2016 Awad A. Bakery. This is an open access article distributed under the Creative Commons Attribution License, which permits unrestricted use, distribution, and reproduction in any medium, provided the original work is properly cited.

\begin{abstract}
We give sufficient conditions on a special space of sequences defined by Mohamed and Bakery (2013) such that the finite rank operators are dense in the complete space of operators whose approximation numbers belong to this sequence space. Hence, under a few conditions, every compact operator would be approximated by finite rank operators. We apply it on the sequence space defined by Tripathy and Mahanta (2003). Our results match those known for $p$-absolutely summable sequences of reals.
\end{abstract}

\section{Introduction and Basic Definitions}

By $\omega$ and $L(V, W)$, we will denote the spaces of all real sequences and all bounded linear operators between two Banach spaces $V$ into $W$, respectively. In [1], Pietsch, by using the approximation numbers and $p$-absolutely summable sequences of real numbers, formed the operator ideals. In [2], Mohamed and Bakery have considered the space $\ell_{M}$, when $M(t)=t^{p}(0<p<\infty)$, which matches especially $\ell^{p}$. A subclass $U$ of $L=\{L(V, W)\}$ is an operator ideal if its components verify the following conditions:

(i) The space $F(V, W)$ of all finite rank operators is a subset of $U(V, W)$.

(ii) The space $U(V, W)$ is linear.

(iii) For two Banach spaces $V_{0}$ and $W_{0}$, if $T \in L\left(V_{0}, V\right), S \in$ $U(V, W)$, and $R \in L\left(W, W_{0}\right)$, then $R S T \in U\left(V_{0}, W_{0}\right)$. See $[3,4]$.

An Orlicz function is a function $M:[0, \infty) \rightarrow[0, \infty)$ which is convex, positive, nondecreasing, and continuous, where $M(0)=0$ and $\lim _{x \rightarrow \infty} M(x)=\infty$. An Orlicz function $M$ is said to satisfy $\Delta_{2}$-condition for all values of $x \geq 0$ if there exists a constant $k>0$, such that $M(2 x) \leq k M(x)$.
Lindenstrauss and Tzafriri [5] used the idea of an Orlicz function to define Orlicz sequence spaces as follows:

$$
\begin{aligned}
\ell_{M} & =\left\{x \in \omega: \exists \lambda>0 \text { with } \rho(\lambda x)=\sum_{k=1}^{\infty} M(|\lambda x(k)|)\right. \\
& <\infty\} .
\end{aligned}
$$

$\left(\ell_{M},\|x\|\right)$ is a Banach space, where $\|x\|=\inf \{\lambda>0$ : $\rho(x / \lambda) \leq 1\}$. The space $\ell^{p}$ is an Orlicz sequence space with $M(x)=x^{p}$ for $1 \leq p<\infty$.

Remark 1. For any Orlicz function $M$, we have $M(\lambda x) \leq$ $\lambda M(x)$, for all $\lambda$ with $0<\lambda<1$.

Let $P_{s}$ be the class of all subsets of $\mathbb{N}=\{0,1,2, \ldots\}$ that do not contain more than $s$ number of elements and let $\left\{\phi_{n}\right\}$ be a nondecreasing sequence of positive reals such that $n \phi_{n+1} \leq$ $(n+1) \phi_{n}$, for all $n \in \mathbb{N}$. Tripathy and Mahanta [6] defined and studied the following sequence space:

$$
\begin{aligned}
& m(\phi, M)=\left\{x=\left(x_{k}\right) \in \omega: \exists \zeta\right. \\
& \left.>0 \text { with } \sup _{s \geq 1, \sigma \in P_{s}} \frac{1}{\phi_{s}} \sum_{k \in \sigma} M\left(\frac{\left|x_{k}\right|}{\zeta}\right)<\infty\right\},
\end{aligned}
$$


with the norm

$$
\rho(x)=\inf \left\{\zeta>0: \sup _{s \geq 1, \sigma \in P_{s}} \frac{1}{\phi_{s}} \sum_{k \in \sigma} M\left(\frac{\left|x_{k}\right|}{\zeta}\right) \leq 1\right\} .
$$

Lemma 2. (i) $\ell_{M} \subseteq m(\phi, M)$.

(ii) $\ell_{M}=m(\phi, M)$ if and only if $\sup _{s} \phi_{s}<\infty$.

As of late, different classes of sequences have been presented using Orlicz functions by Braha [7], Raj and Sharma Sunil [8], Raj et al. [9], and many others ([10-13]).

Definition 3 (see $[14,15]$ ). A special space of sequences (sss) is a linear space $E$ with the following:

(1) $e_{n} \in E$ for all $n \in \mathbb{N}$, where $e_{n}=\{0,0, \ldots, 1,0,0, \ldots\}$ with 1 appearing at $n$th place for all $n \in \mathbb{N}$.

(2) $E$ is solid".

(3) $\left(x_{0}, x_{0}, x_{1}, x_{1}, \ldots\right) \in E$, if $\left(x_{n}\right)_{n \in \mathbb{N}} \in E$.

A premodular (sss) $E_{\rho}$ is a (sss) and there is a function $\rho$ : $E \rightarrow[0, \infty[$ with the following:

(i) $\rho(x) \geq 0$, for each $x \in E$ and $\rho(x)=0 \Leftrightarrow x=\theta$, where $\theta$ is the zero element of $E$.

(ii) $\rho$ satisfies $\Delta_{2}$-condition.

(iii) For each $x, y \in E, \rho(x+y) \leq k(\rho(x)+\rho(y))$ holds for some $k \geq 1$.

(iv) The space $E$ is $\rho$-solid; that is, $\rho\left(\left(x_{n}\right)\right) \leq \rho\left(\left(y_{n}\right)\right)$, whenever $\left|x_{n}\right| \leq\left|y_{n}\right|$, for all $n \in \mathbb{N}$.

(v) For some numbers $k_{0} \geq 1$, the inequality $\rho\left(\left(x_{n}\right)_{n \in \mathbb{N}}\right) \leq$ $\rho\left(\left(x_{0}, x_{0}, x_{1}, x_{1}, \ldots\right)\right) \leq k_{0} \rho\left(\left(x_{n}\right)_{n \in \mathbb{N}}\right)$ holds.

(vi) $\bar{F}=E_{\rho}$; that is, the set of all finite sequences $F$ is $\rho$ dense in $E$.

(vii) For each $\lambda>0$, there is a constant $\xi>0$ such that $\rho(\lambda, 0,0,0, \ldots) \geq \xi \lambda \rho(1,0,0,0, \ldots)$.

Condition (ii) says that $\rho$ is continuous at $\theta$. The function $\rho$ defines a metrizable topology in $E$ and the linear space $E$ enriched with this topology is denoted by $E_{\rho}$.

Definition 4 (see [16]). Consider the following:

$$
U_{E}^{\text {app }}:=\left\{U_{E}^{\text {app }}(V, W)\right\}
$$

where

$$
U_{E}^{\text {app }}(V, W):=\left\{T \in L(V, W):\left(\alpha_{n}(T)\right)_{n \in \mathbb{N}} \in E\right\} .
$$

Theorem 5 (see [2]). If $E$ is a (sss), then $U_{E}^{a p p}$ is an operator ideal.

We explain some results related to the operator spaces.

\section{Main Results}

In this part, we give sufficient conditions on $E$ such that the finite rank operators are dense in the complete space of operators $U_{E}^{\text {app }}(V, W)$.

Lemma 6. If $E_{\rho}$ is a premodular (sss) and $\left(x_{n}\right) \in E_{\rho}$ is a decreasing sequence of positive reals, then

$$
\begin{aligned}
& \rho(\overbrace{0,0,0, \ldots, 0}^{2 n}, x_{n}, x_{n+1}, x_{n+2}, \ldots) \\
& \quad \leq k_{0} \rho(\overbrace{0,0,0, \ldots, 0}^{n}, x_{n}, x_{n+1}, x_{n+2}, \ldots) .
\end{aligned}
$$

Proof. By using Definition 3, conditions (iv) and (v), and since the elements of $E$ are decreasing, we get

$$
\begin{aligned}
& \rho(\overbrace{0,0,0, \ldots, 0}^{2 n}, x_{n}, x_{n+1}, x_{n+2}, \ldots) \\
& \quad \leq \rho(\overbrace{0,0,0, \ldots, 0}^{2 n}, x_{n}, x_{n}, x_{n+1}, x_{n+1}, \ldots) \\
& \quad \leq k_{0} \rho(\overbrace{0,0,0, \ldots, 0}^{n}, x_{n}, x_{n+1}, x_{n+2}, \ldots) .
\end{aligned}
$$

Theorem 7. Let $E_{\rho}$ be a premodular (sss); then $\overline{F(V, W)^{g}}=$ $U_{E_{\rho}}^{a p p}(V, W)$, where $g(T)=\rho\left(\alpha_{n}(T)_{n \in \mathbb{N}}\right)$.

Proof. To prove that $F(V, W) \subseteq U_{E}^{\text {app }}(V, W)$, since $e_{m} \in E$ for each $m \in \mathbb{N}$, from the linearity of $E$ and $T \in F(V, W)$, then finitely many elements of $\left(\alpha_{n}(T)\right)_{n \in \mathbb{N}}$ are different from zero. Hence, $T \in U_{E}^{\mathrm{app}}(V, W)$. For the other inclusion $U_{E}^{\mathrm{app}}(V, W) \subseteq$ $\overline{F(V, W)}$, let $T \in U_{E}^{\text {app }}(V, W)$ and, from the definition of approximation numbers, there is $N \in \mathbb{N}, N>0, A_{N}$ with $\operatorname{rank}\left(A_{N}\right) \leq N$ and also

$$
\left\|T-A_{N}\right\| \leq 2 \alpha_{N}(T) .
$$

Since $\alpha_{N}(T) \rightarrow 0$ as $N \rightarrow \infty$, then $\left\|T-A_{N}\right\| \rightarrow 0$ as $N \rightarrow \infty$; we have to prove that $\rho\left(\left(\alpha_{n}\left(T-A_{N}\right)\right)_{n \in \mathbb{N}}\right) \rightarrow 0$ as 
$N \rightarrow \infty$, by taking $N=8 \eta$, where $\eta$ is a natural number. From Definition 3, condition (iii), we have

$$
\begin{aligned}
& d\left(T, A_{N}\right)=\rho\left(\left(\alpha_{n}\left(T-A_{N}\right)\right)_{n \in \mathbb{N}}\right) \\
& =\rho\left[\left(\alpha_{0}\left(T-A_{N}\right), \alpha_{1}\left(T-A_{N}\right), \ldots,\right.\right. \\
& \left.\alpha_{8 \eta-1}\left(T-A_{N}\right), 0,0,0, \ldots\right)+(\overbrace{0,0,0, \ldots, 0}^{8 \eta}, \\
& \alpha_{8 \eta}\left(T-A_{N}\right), \alpha_{8 \eta+1}\left(T-A_{N}\right), \ldots, \alpha_{12 \eta-1}\left(T-A_{N}\right), \\
& 0,0,0, \ldots)+(\overbrace{0,0,0, \ldots, 0}^{12 \eta} \alpha_{12 \eta}\left(T-A_{N}\right), \\
& \left.\left.\alpha_{12 \eta+1}\left(T-A_{N}\right), \ldots\right)\right] \leq k^{2}\left[\rho \left(\alpha_{0}\left(T-A_{N}\right),\right.\right. \\
& \left.\alpha_{1}\left(T-A_{N}\right), \ldots, \alpha_{8 \eta-1}\left(T-A_{N}\right), 0,0,0, \ldots\right) \\
& +\rho(\overbrace{0,0,0, \ldots, 0, \alpha_{8 \eta}}^{8 \eta}\left(T-A_{N}\right), \alpha_{8 \eta+1}\left(T-A_{N}\right), \\
& \left.\quad \ldots, \alpha_{12 \eta-1}\left(T-A_{N}\right), 0,0,0, \ldots\right)+\rho(\overbrace{0,0,0, \ldots, 0}^{12 \eta}, \\
& \left.\left.\quad \alpha_{12 \eta}\left(T-A_{N}\right), \alpha_{12 \eta+1}\left(T-A_{N}\right), \ldots\right)\right]=k^{2}\left[I_{1}(N)\right. \\
& \left.+I_{2}(\eta)+I_{3}(\eta)\right] .
\end{aligned}
$$

Since $\alpha_{n}\left(A_{N}\right)=0$ for $n \geq N$, then

$$
\alpha_{n}\left(T-A_{N}\right) \leq \alpha_{n-N}(T) .
$$

By using Lemma 6, inequality (10), and Definition 3, condition (v), we get

$$
\begin{aligned}
I_{3}(\eta)=\rho(\overbrace{0,0,0, \ldots, 0}^{12 \eta}, \alpha_{12 \eta}\left(T-A_{N}\right), \\
\left.\alpha_{12 \eta+1}\left(T-A_{N}\right), \ldots\right) \leq \rho(\overbrace{0,0,0, \ldots, 0}^{12 \eta}, \alpha_{4 \eta}(T), \\
\left.\alpha_{4 \eta+1}(T), \ldots\right) \leq k_{0} \rho(\overbrace{0,0,0, \ldots, 0}^{6 \eta} \alpha_{4 \eta}(T), \\
\left.\alpha_{4 \eta+1}(T), \ldots\right) \leq k_{0}^{2} \rho(\overbrace{0,0,0, \ldots, 0}^{3 \eta}, \alpha_{4 \eta}(T), \\
\left.\alpha_{4 \eta+1}(T), \ldots\right) \leq k_{0}^{2} \rho(\overbrace{0,0,0, \ldots, 0}^{3 \eta}, \alpha_{3 \eta}(T), \\
\left.\alpha_{3 \eta+1}(T), \ldots\right)=k_{0}^{2} \rho\left(\left(\alpha_{n}(T)\right)_{n=3 \eta}^{\infty}\right) \longrightarrow 0
\end{aligned}
$$

Now, using Lemma 6 and Definition 3, condition (v), we have

$$
\begin{aligned}
& I_{2}(\eta)=\rho(\overbrace{0,0,0, \ldots, 0}^{8 \eta}, \alpha_{8 \eta}\left(T-A_{N}\right), \\
& \left.\quad \alpha_{8 \eta+1}\left(T-A_{N}\right), \ldots, \alpha_{12 \eta-1}\left(T-A_{N}\right), 0,0,0, \ldots\right) \\
& \quad \leq k_{0} \rho(\overbrace{0,0,0, \ldots, 0, \alpha_{8 \eta}}^{4 \eta}\left(T-A_{N}\right), \\
& \left.\quad \alpha_{8 \eta+1}\left(T-A_{N}\right), \ldots, \alpha_{12 \eta-1}\left(T-A_{N}\right), 0,0,0, \ldots\right) \\
& \quad \leq k_{0} \rho\left(\alpha_{0}\left(T-A_{N}\right), \alpha_{1}\left(T-A_{N}\right), \ldots,\right. \\
& \left.\quad \alpha_{8 \eta-1}\left(T-A_{N}\right), 0,0,0, \ldots\right)=k_{0} I_{1}(N) .
\end{aligned}
$$

Finally, we have to show that $I_{1}(N) \rightarrow 0$ as $N \rightarrow \infty$. Since $T \epsilon$ $U_{E}^{\text {app }}(V, W)$ and $\rho$ is continuous at $\theta$, we have $\rho\left(\alpha_{k}(T)\right)_{k=n}^{\infty} \rightarrow 0$ as $n \rightarrow \infty$. Then, for each $\varepsilon>0$, there exists $N_{0}(\varepsilon)$ such that for all $n \geq N_{0}(\varepsilon)$ we have

$$
\rho\left(\left(\alpha_{k}(T)\right)_{k=n}^{\infty}\right)<\varepsilon .
$$

By taking $\varepsilon_{1}=\varepsilon / 3 l k$ for each $n \geq N_{0}\left(\varepsilon_{1}\right)$ and using inequality (13) and Definition 3, conditions (ii) and (iii), then we have

$$
\begin{aligned}
& I_{1}(N)=\rho\left(\alpha_{0}\left(T-A_{N}\right), \alpha_{1}\left(T-A_{N}\right), \ldots, \alpha_{N-1}(T\right. \\
& \left.\left.-A_{N}\right), 0,0,0, \ldots\right) \leq k\left[\rho \left(\alpha_{0}\left(T-A_{N}\right),\right.\right. \\
& \left.\alpha_{1}\left(T-A_{N}\right), \ldots, \alpha_{N_{0}-1}\left(T-A_{N}\right), 0,0,0, \ldots\right) \\
& +\rho(\overbrace{0,0,0, \ldots, 0}^{N_{0}} \alpha_{N_{0}}\left(T-A_{N}\right), \alpha_{N_{0}+1}\left(T-A_{N}\right), \\
& \left.\left.\ldots, \alpha_{N-1}\left(T-A_{N}\right), 0,0,0, \ldots\right)\right] \leq k\left[\rho \left(\left\|T-A_{N}\right\|,\right.\right. \\
& \left.\left\|T-A_{N}\right\|, \ldots,\left\|T-A_{N}\right\|, 0,0,0, \ldots\right) \\
& +\rho(\overbrace{0,0,0, \ldots, 0,2 \alpha_{N}(T), 2 \alpha_{N}(T), \ldots, 2 \alpha_{N}(T),}^{N_{0}} \\
& 0,0,0, \ldots)] \leq k\left[\left\|T-A_{N}\right\| l \rho(\overbrace{1,1,1, \ldots, 1}^{N_{0}}, 0,0,0,\right. \\
& \ldots)+2 l \rho(\overbrace{0,0,0, \ldots, 0, \alpha_{N_{0}}}^{N_{0}}(T), \alpha_{N_{0}+1}(T), \ldots, \\
& \left.\left.\quad \alpha_{N}(T), 0,0,0, \ldots\right)\right] \leq k\left[\left\|T-A_{N}\right\| l k_{1}(\varepsilon)+2 l \varepsilon_{1}\right],
\end{aligned}
$$

where $k_{1}(\varepsilon)=\rho(\overbrace{1,1,1, \ldots, 1}^{N_{0}}, 0,0,0, \ldots)$, and since $\| T-$ $A_{N} \| \rightarrow 0$ as $N \rightarrow \infty$, then for each $\varepsilon>0$ there exists $N$ 
such that $\left\|T-A_{N}\right\| k_{1}(\varepsilon) \leq \varepsilon_{1}$, for that we have $I_{1}(N) \leq$ $k\left[l \varepsilon_{1}+2 l \varepsilon_{1}\right]=3 k l \varepsilon_{1}=\varepsilon$. This completes the proof.

We give here the sufficient conditions on the sequence spaces $m(\phi, M)$ such that the class of all bounded linear operators between any arbitrary Banach spaces with $\left(\alpha_{n}(T)\right)_{n \in \mathbb{N}}$ in these sequence spaces form an ideal operator; the ideal of the finite rank operators in the class of Banach spaces is dense in $U_{m(\phi, M)}^{\text {app }}(V, W)$.

Theorem 8. Let $M$ be an Orlicz function satisfying $\Delta_{2}-$ condition. Then,
(a) $U_{m(\phi, M)}^{a p p}$ is an operator ideal,
(b) $\overline{F(V, W)}=U_{m(\phi, M)}^{a p p}(V, W)$.

Proof. We first prove that the space $m(\phi, M)$ is a (sss).

(1) Let $\lambda_{1}, \lambda_{2} \in \mathbb{R}$ and $x, y \in m(\phi, M)$; then there exist $\zeta_{1}>0$ and $\zeta_{2}>0$ such that

$$
\begin{aligned}
& \sup _{s \geq 1, \sigma \in P_{s}} \frac{1}{\phi_{s}} \sum_{k \in \sigma} M\left(\frac{\left|x_{k}\right|}{\zeta_{1}}\right)<\infty, \\
& \sup _{s \geq 1, \sigma \in P_{s}} \frac{1}{\phi_{s}} \sum_{k \in \sigma} M\left(\frac{\left|x_{k}\right|}{\zeta_{2}}\right)<\infty .
\end{aligned}
$$

Let $\zeta_{3}=\max \left(2\left|\lambda_{1}\right| \zeta_{1}, 2\left|\lambda_{2}\right| \zeta_{2}\right)$. Since $M$ is nondecreasing convex function with $\Delta_{2}$-condition, we have

$$
\begin{aligned}
& \sum_{k \in \sigma} M\left(\frac{\left|\lambda_{1} x_{k}+\lambda_{2} y_{k}\right|}{\zeta_{3}}\right) \\
& \quad \leq \frac{1}{2}\left[\sum_{k \in \sigma} M\left(\frac{\left|x_{k}\right|}{\zeta_{1}}\right)+\sum_{k \in \sigma} M\left(\frac{\left|y_{k}\right|}{\zeta_{2}}\right)\right] .
\end{aligned}
$$

So, we get

$$
\begin{gathered}
\sup _{s \geq 1, \sigma \in P_{s}} \frac{1}{\phi_{s}} \sum_{k \in \sigma} M\left(\frac{\left|\lambda_{1} x_{k}+\lambda_{2} y_{k}\right|}{\zeta_{3}}\right) \\
\leq \frac{1}{2}\left[\sup _{s \geq 1, \sigma \in P_{s}} \frac{1}{\phi_{s}} \sum_{k \in \sigma} M\left(\frac{\left|x_{k}\right|}{\zeta_{1}}\right)\right. \\
\left.+\sup _{s \geq 1, \sigma \in P_{s}} \frac{1}{\phi_{s}} \sum_{k \in \sigma} M\left(\frac{\left|y_{k}\right|}{\zeta_{2}}\right)\right] .
\end{gathered}
$$

Thus, $\lambda_{1} x+\lambda_{2} y \in m(\phi, M)$. Hence, $m(\phi, M)$ is a linear space over the field of real numbers. Also, since $e_{n} \in \ell_{M}$ and $\ell_{M} \subseteq$ $m(\phi, M)$, we have $e_{n} \in m(\phi, M)$ for all $n \in \mathbb{N}$.

(2) Let $x \in \omega$ and $y=\left(y_{k}\right)_{k=0}^{\infty} \in m(\phi, M)$ with $\left|x_{k}\right| \leq\left|y_{k}\right|$ for each $k \in \mathbb{N}$; since $M$ is nondecreasing, then we get

$$
\begin{aligned}
& \sup _{s \geq 1, \sigma \in P_{s}} \frac{1}{\phi_{s}} \sum_{k \in \sigma} M\left(\frac{\left|x_{k}\right|}{\zeta}\right) \leq \sup _{s \geq 1, \sigma \in P_{s}} \frac{1}{\phi_{s}} \sum_{k \in \sigma} M\left(\frac{\left|y_{k}\right|}{\zeta}\right) \\
& \quad<\infty
\end{aligned}
$$

then $x=\left(x_{k}\right)_{k=0}^{\infty} \in m(\phi, M)$.
(3) Let $x=\left(x_{k}\right)_{k=0}^{\infty} \in m(\phi, M)$; then we have

$$
\begin{aligned}
& \sup _{s \geq 1, \sigma \in P_{s}} \frac{1}{\phi_{s}} \sum_{k \in \sigma} M\left(\frac{\left|x_{[k / 2]}\right|}{\zeta}\right) \\
& \leq 2 \sup _{s \geq 1, \sigma \in P_{s}} \frac{1}{\phi_{s}} \sum_{k \in \sigma} M\left(\frac{\left|x_{k}\right|}{\zeta}\right)<\infty ;
\end{aligned}
$$

then $x=\left(x_{[k / 2]}\right)_{k=0}^{\infty} \in m(\phi, M)$.

Finally, we have proved that the space $m(\phi, M)$ with $\rho(x)$ is a premodular (sss). $x=\theta$.

(i) Clearly, $\rho(x) \geq 0$ for all $x \in m(\phi, M)$ and $\rho(x)=0 \Leftrightarrow$

(ii) Let $\lambda \in \mathbb{R}$ and $x \in m(\phi, M)$; then for $\lambda \neq 0$ we have

$\rho(\lambda x)$

$$
\begin{aligned}
& =\inf \left\{\zeta>0: \sup _{s \geq 1, \sigma \in P_{s}} \frac{1}{\phi_{s}} \sum_{k \in \sigma} M\left(\frac{\left|\lambda x_{k}\right|}{\zeta}\right) \leq 1\right\} \\
& =\inf \left\{|\lambda| \mu>0: \sup _{s \geq 1, \sigma \in P_{s}} \frac{1}{\phi_{s}} \sum_{k \in \sigma} M\left(\frac{\left|x_{k}\right|}{\mu}\right) \leq 1\right\},
\end{aligned}
$$

where $\mu=\zeta /|\lambda|$. Thus, $\rho(\lambda x)=|\lambda| \inf \{\mu>0$ : $\left.\sup _{s \geq 1, \sigma \in P_{s}}\left(1 / \phi_{s}\right) \sum_{k \in \sigma} M\left(\left|x_{k}\right| / \mu\right) \leq 1\right\}=|\lambda| \rho(x)$.

Also, for $\lambda=0$, we have $\rho(\lambda x)=\lambda \rho(x)=0$.

(iii) Let $x, y \in m(\phi, M)$; then there exist $\zeta_{1}>0$ and $\zeta_{2}>0$ such that

$$
\begin{aligned}
& \sup _{s \geq 1, \sigma \in P_{s}} \frac{1}{\phi_{s}} \sum_{k \in \sigma} M\left(\frac{\left|x_{k}\right|}{\zeta_{1}}\right) \leq 1, \\
& \sup _{s \geq 1, \sigma \in P_{s}} \frac{1}{\phi_{s}} \sum_{k \in \sigma} M\left(\frac{\left|x_{k}\right|}{\zeta_{2}}\right) \leq 1 .
\end{aligned}
$$

Let $\zeta=\zeta_{1}+\zeta_{2}$, and since $M$ is nondecreasing and convex, then we have

$$
\begin{aligned}
& \sup _{s \geq 1, \sigma \in P_{s}} \frac{1}{\phi_{s}} \sum_{k \in \sigma} M\left(\frac{\left|x_{k}+y_{k}\right|}{\zeta}\right) \leq \sup _{s \geq 1, \sigma \in P_{s}} \frac{1}{\phi_{s}} \\
& \cdot \sum_{k \in \sigma} M\left(\frac{\left|x_{k}\right|+\left|y_{k}\right|}{\zeta_{1}+\zeta_{2}}\right) \leq \sup _{s \geq 1, \sigma \in P_{s}} \frac{1}{\phi_{s}} \\
& \cdot \sum_{k \in \sigma}\left[\left(\frac{\zeta_{1}}{\zeta_{1}+\zeta_{2}}\right) M\left(\frac{\left|x_{k}\right|}{\zeta_{1}}\right)\right. \\
& \left.+\left(\frac{\zeta_{2}}{\zeta_{1}+\zeta_{2}}\right) M\left(\frac{\left|y_{k}\right|}{\zeta_{2}}\right)\right] \leq\left(\frac{\zeta_{1}}{\zeta_{1}+\zeta_{2}}\right)_{s \geq 1, \sigma \in P_{s}} \frac{1}{\phi_{s}} \\
& \quad \cdot \sum_{k \in \sigma} M\left(\frac{\left|x_{k}\right|}{\zeta_{1}}\right)+\left(\frac{\zeta_{2}}{\zeta_{1}+\zeta_{2}}\right) \sum_{k \in \sigma} M\left(\frac{\left|y_{k}\right|}{\zeta_{2}}\right) \leq 1 .
\end{aligned}
$$


Since $\zeta$ 's are nonnegative, we have

$$
\begin{aligned}
\rho(x+y) & \inf \left\{\zeta>0: \sup _{s \geq 1, \sigma \in P_{s}} \frac{1}{\phi_{s}} \sum_{k \in \sigma} M\left(\frac{\left|x_{k}+y_{k}\right|}{\zeta}\right) \leq 1\right\} \\
\leq & \inf \left\{\zeta_{1}>0: \sup _{s \geq 1, \sigma \in P_{s}} \frac{1}{\phi_{s}} \sum_{k \in \sigma} M\left(\frac{\left|x_{k}\right|}{\zeta_{1}}\right) \leq 1\right\} \\
& +\inf \left\{\zeta_{2}>0: \sup _{s \geq 1, \sigma \in P_{s}} \frac{1}{\phi_{s}} \sum_{k \in \sigma} M\left(\frac{\left|y_{k}\right|}{\zeta_{2}}\right) \leq 1\right\} \\
= & \rho(x)+\rho(y) .
\end{aligned}
$$

(iv) Let $\left|x_{k}\right| \leq\left|y_{k}\right|$ for each $k \in \mathbb{N}$, and since $M$ is nondecreasing, then we get

$$
\sup _{s \geq 1, \sigma \in P_{s}} \frac{1}{\phi_{s}} \sum_{k \in \sigma} M\left(\frac{\left|x_{k}\right|}{\zeta}\right) \leq \sup _{s \geq 1, \sigma \in P_{s}} \frac{1}{\phi_{s}} \sum_{k \in \sigma} M\left(\frac{\left|y_{k}\right|}{\zeta}\right)
$$

thus,

$$
\begin{aligned}
& \inf \left\{\zeta>0: \sup _{s \geq 1, \sigma \in P_{s}} \frac{1}{\phi_{s}} \sum_{k \in \sigma} M\left(\frac{\left|x_{k}\right|}{\zeta}\right)\right\} \\
& \quad \leq \inf \left\{\zeta>0: \sup _{s \geq 1, \sigma \in P_{s}} \frac{1}{\phi_{s}} \sum_{k \in \sigma} M\left(\frac{\left|y_{k}\right|}{\zeta}\right)\right\} .
\end{aligned}
$$

So, $\rho(x) \leq \rho(y)$.

(v) Since

$$
\begin{aligned}
& \sup _{s \geq 1, \sigma \in P_{s}} \frac{1}{\phi_{s}} \sum_{k \in \sigma} M\left(\frac{\left|x_{[k / 2]}\right|}{\zeta}\right) \\
& \leq 2 \sup _{s \geq 1, \sigma \in P_{s}} \frac{1}{\phi_{s}} \sum_{k \in \sigma} M\left(\frac{\left|x_{k}\right|}{\zeta}\right),
\end{aligned}
$$

we have

$$
\begin{aligned}
& \inf \left\{\zeta>0: \sup _{s \geq 1, \sigma \in P_{s}} \frac{1}{\phi_{s}} \sum_{k \in \sigma} M\left(\frac{\left|x_{[k / 2]}\right|}{\zeta}\right)\right\} \\
& \quad \leq 2 \inf \left\{\zeta>0: \sup _{s \geq 1, \sigma \in P_{s}} \frac{1}{\phi_{s}} \sum_{k \in \sigma} M\left(\frac{\left|x_{k}\right|}{\zeta}\right)\right\} .
\end{aligned}
$$

So, $\rho\left(\left(x_{k}\right)\right) \leq \rho\left(\left(x_{[k / 2]}\right)\right) \leq 2 \rho\left(\left(x_{k}\right)\right)$.

(vi) For each $x=\left(x_{k}\right)_{k=0}^{\infty} \in m(\phi, M)$, then

$$
\begin{aligned}
& \rho\left(\left(x_{k}\right)_{k=0}^{\infty}\right) \\
& \quad=\inf \left\{\zeta>0: \sup _{s \geq 1, \sigma \in P_{s}} \frac{1}{\phi_{s}} \sum_{k \in \sigma} M\left(\frac{\left|x_{[k / 2]}\right|}{\zeta}\right)<\infty\right\} ;
\end{aligned}
$$

we can find $t \in \mathbb{N}$ such that $\rho\left(\left(x_{k}\right)_{k=t}^{\infty}\right)<\infty$. This means the set of all finite sequences is $\rho$-dense in $m(\phi, M)$. (vii) For any $\lambda>0$, there exists a constant $\zeta \in] 0,1]$ such that

$$
\rho(\lambda, 0,0,0, \ldots) \geq \zeta \lambda \rho(1,0,0,0, \ldots) .
$$

By using Theorems 5 and 7, the proof follows.

As special cases of the above theorem, we obtain the following corollaries.

Corollary 9. If $\sup _{s} \phi_{s}<\infty$, one gets that
(a) $U_{\ell_{M}}^{a p p}$ is an operator ideal,
(b) $\overline{F(V, W)}=U_{\ell_{M}}^{a p p}(V, W)$.

Corollary 10. If $\sup _{s} \phi_{s}<\infty$ and $M(t)=t^{p}$ with $0<p<\infty$, one gets that
(a) $U_{\ell^{p}}^{a p p}$ is an operator ideal,
(b) $\overline{F(V, W)}=U_{\ell^{p}}^{a p p}(V, W)$. See [1].

Theorem 11. If $E_{\rho}$ is a premodular (sss), then $U_{E_{\rho}}^{a p p}(V, W)$ is complete.

Proof. Let $\left(T_{m}\right)$ be a Cauchy sequence in $U_{E_{\rho}}^{\text {app }}(V, W)$; then, by using Definition 3, condition (vii), and since $U_{E_{\rho}}^{\text {app }}(V, W) \subseteq$ $L(V, W)$, we get

$$
\begin{aligned}
& \rho\left(\left(\alpha_{n}\left(T_{i}-T_{j}\right)\right)_{n \in \mathbb{N}}\right) \geq \rho\left(\alpha_{0}\left(T_{i}-T_{j}\right), 0,0,0, \ldots\right) \\
& \quad=\rho\left(\left\|T_{i}-T_{j}\right\|, 0,0,0, \ldots\right) \\
& \quad \geq \xi\left\|T_{i}-T_{j}\right\| \rho(1,0,0,0, \ldots) ;
\end{aligned}
$$

then $\left(T_{m}\right)$ is also a Cauchy sequence in $L(V, W)$. Since the space $L(V, W)$ is a Banach space, then there exists $T \in$ $L(V, W)$ such that $\left\|T_{m}-T\right\| \rightarrow 0$, as $m \rightarrow \infty$, and since $\left(\alpha_{n}\left(T_{m}\right)\right)_{n \in \mathbb{N}} \in E$, for each $m \in \mathbb{N}$, then from Definition 3, conditions (iii) and (v), and since $\rho$ is continuous at $\theta$, we have

$$
\begin{aligned}
\rho\left(\left(\alpha_{n}(T)\right)_{n \in \mathbb{N}}\right)= & \rho\left(\alpha_{n}\left(T-T_{m}+T_{m}\right)\right)_{n \in \mathbb{N}} \\
\leq & k \rho\left(\alpha_{[n / 2]}\left(T-T_{m}\right)\right)_{n \in \mathbb{N}} \\
& +k \rho\left(\alpha_{[n / 2]}\left(T_{m}\right)\right)_{n \in \mathbb{N}} \\
\leq & k \rho\left(\left\|T_{m}-T\right\|\right)_{n \in \mathbb{N}} \\
& +k \rho\left(\alpha_{n}\left(T_{m}\right)\right)_{n \in \mathbb{N}}<\varepsilon ;
\end{aligned}
$$

we get $\left(\alpha_{n}(T)\right)_{n \in \mathbb{N}} \in E$, and then $T \in U_{E_{\rho}}^{\text {app }}(V, W)$. This finishes the proof.

By applying Theorem 11 on $m(\phi, M)$, we can easily conclude the next corollaries.

Corollary 12. Pick up an Orlicz function $M$ which satisfies $\Delta_{2}$-condition. Then, $M$ is continuous from the right at 0 and $U_{m(\phi, M)}^{a p p}(V, W)$ is complete. 
Corollary 13. Pick up an Orlicz function $M$ which satisfies $\Delta_{2}$ condition with $\sup _{s} \phi_{s}<\infty$. Then, $M$ is continuous from the right at 0 and $U_{l_{M}}^{a p p}(X, Y)$ is complete.

Corollary 14. $U_{\ell^{p}}^{a p p}(V, W)$ is complete if $M(t)=t^{p}$ and $p \in$ $(0, \infty)$.

\section{Competing Interests}

The author declares that he has no competing interests.

\section{References}

[1] A. Pietsch, Operator Ideals, vol. 20, North-Holland, Amsterdam, The Netherlands, 1980.

[2] N. F. Mohamed and A. A. Bakery, "Mappings of type Orlicz and generalized Cesáro sequence space," Journal of Inequalities and Applications, vol. 2013, article 186, 2013.

[3] N. J. Kalton, "Spaces of compact operators," Mathematische Annalen, vol. 208, pp. 267-278, 1974.

[4] Å. Lima and E. Oja, "Ideals of finite rank operators, intersection properties of balls, and the approximation property," Studia Mathematica, vol. 133, no. 2, pp. 175-186, 1999.

[5] J. Lindenstrauss and L. Tzafriri, "On Orlicz sequence spaces," Israel Journal of Mathematics, vol. 10, pp. 379-390, 1971.

[6] B. C. Tripathy and S. Mahanta, "On a class of sequences related to the $\ell_{p}$ space defined by Orlicz functions," Soochow Journal of Mathematics, vol. 29, pp. 379-391, 2003.

[7] N. L. Braha, "A new class of sequences related to the $l_{p}$ spaces defined by sequences of Orlicz functions," Journal of Inequalities and Applications, vol. 2011, Article ID 539745, 10 pages, 2011.

[8] K. Raj and K. Sharma Sunil, "Some difference sequence spaces defined by Musielak-Orlicz functions," Mathematica Pannonica, vol. 24, pp. 33-43, 2013.

[9] K. Raj, S. Jamwal, and S. K. Sharma, "New classes of generalized sequence spaces defined by an Orlicz function," Journal of Computational Analysis and Applications, vol. 15, no. 4, pp. 730737, 2013.

[10] B. C. Tripathy and S. Borgogain, "The sequence space $m\left(M, \emptyset, \triangle_{m}^{n}, p\right)^{F}$, Mathematical Modelling and Analysis, vol. 13, no. 4, pp. 577-586, 2008.

[11] B. C. Tripathy and H. Dutta, "On some new paranormed difference sequence spaces defined by Orlicz functions," Kyungpook Mathematical Journal, vol. 50, no. 1, pp. 59-69, 2010.

[12] B. C. Tripathy and B. Hazarika, "Some I-convergent sequence spaces defined by Orlicz functions," Acta Mathematicae Applicatae Sinica, vol. 27, no. 1, pp. 149-154, 2011.

[13] B. C. Tripathy and B. Sarma, "Vector valued double sequence spaces defined by orlicz function," Mathematica Slovaca, vol. 59, no. 6, pp. 767-776, 2009.

[14] A. A. Bakery, "Mappings of type generalized de La Vallée Poussin's mean," Journal of Inequalities and Applications, vol. 2013, article 518, 2013.

[15] A. A. Bakery, "Operator ideal of Cesaro type sequence spaces involving lacunary sequence," Abstract and Applied Analysis, vol. 2014, Article ID 419560, 6 pages, 2014.

[16] A. A. Bakery, "Operator ideal of Norlund-type sequence spaces," Journal of Inequalities and Applications, vol. 2015, article 255, 2015. 


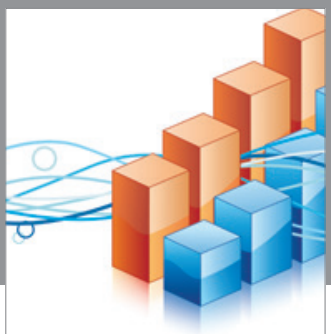

Advances in

Operations Research

vatem alat4

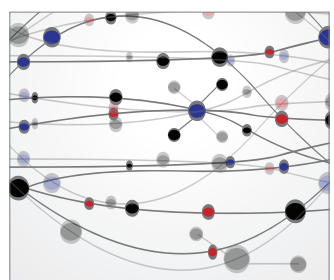

\section{The Scientific} World Journal
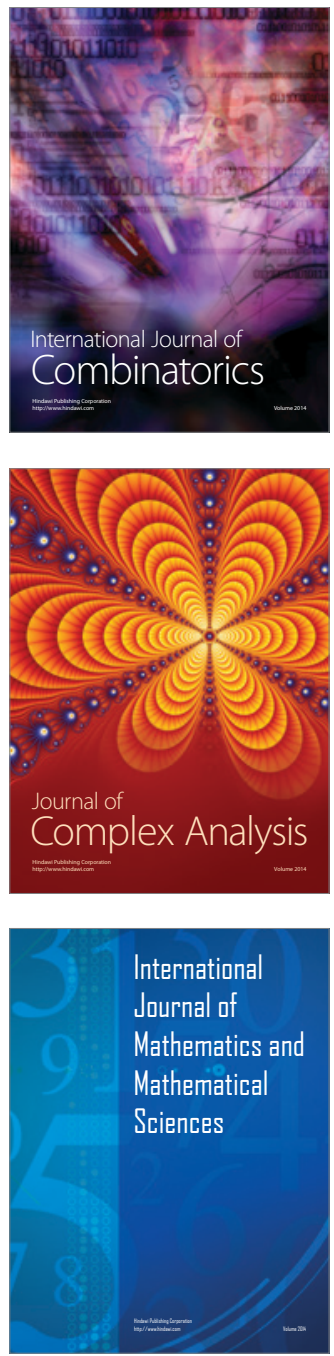
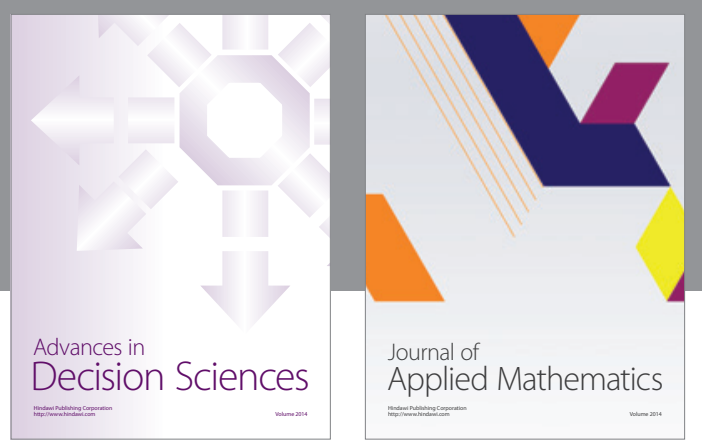

Algebra

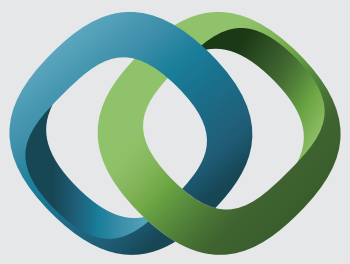

\section{Hindawi}

Submit your manuscripts at

http://www.hindawi.com
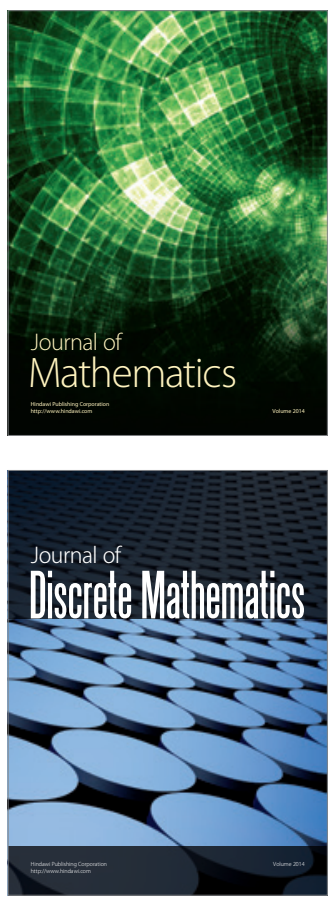

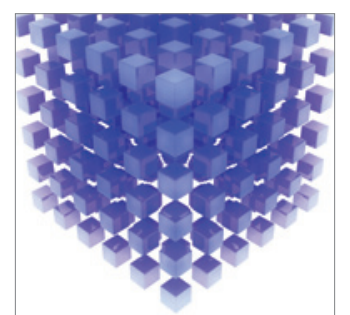

Mathematical Problems in Engineering
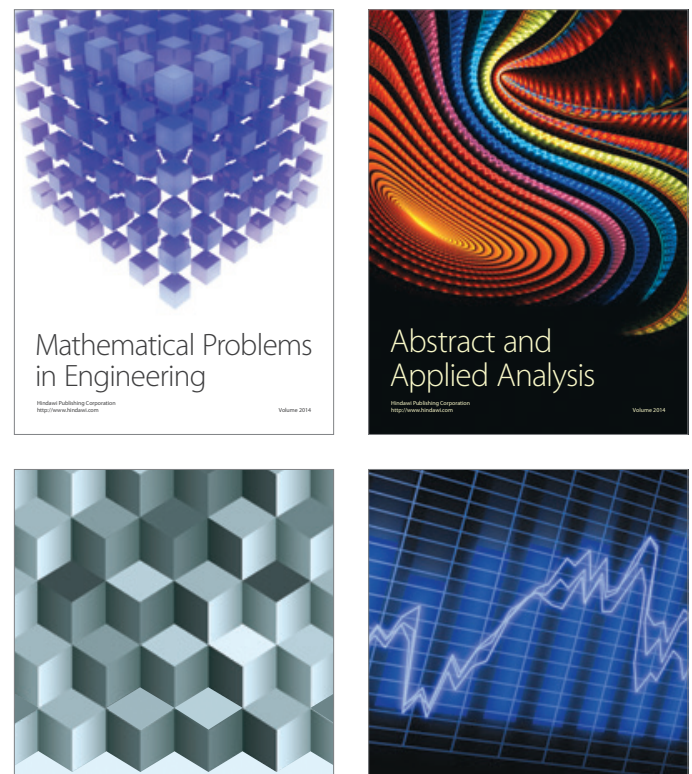

Journal of

Function Spaces

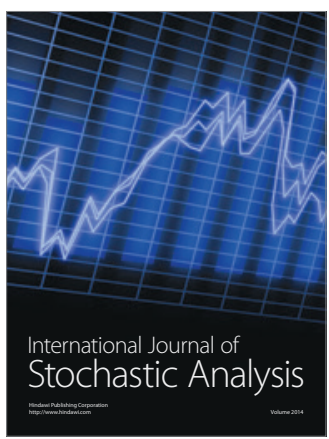

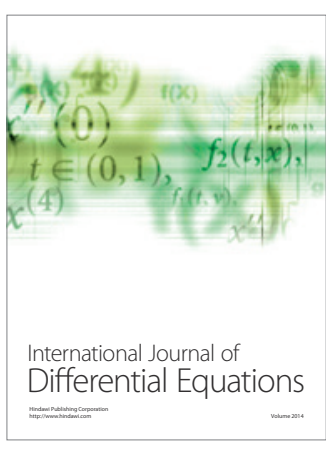
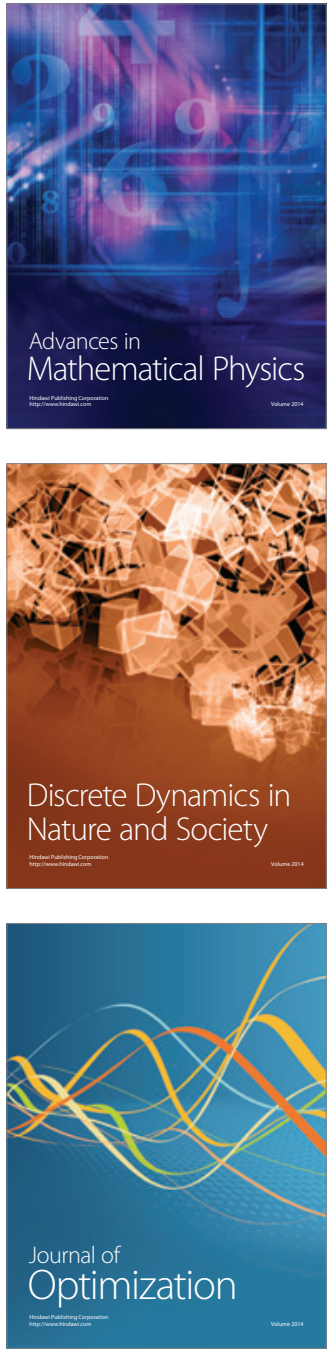